\title{
Stochastic Perron's method and elementary strategies for zero-sum differential games
}

\author{
Mihai Sîrbu * \\ June 14, 2018
}

\begin{abstract}
We develop here the Stochastic Perron Method in the framework of two-player zero-sum differential games. We consider the formulation of the game where both players play, symmetrically, feed-back strategies (as in CR09] and [PZ12]) as opposed to the Elliott-Kalton formulation prevalent in the literature. The class of feed-back strategies we use is carefully chosen so that the state equation admits strong solutions and the technicalities involved in the Stochastic Perron Method carry through in a rather simple way. More precisely, we define the game over elementary strategies, which are well motivated by intuition. Within this framework, the Stochastic Perron Method produces a viscosity sub-solution of the upper Isaacs equation dominating the upper value of the game, and a viscosity super-solution of the upper Isaacs equation lying below the upper value of the game. Using a viscosity comparison result we obtain that the upper value is the unique and continuous viscosity solution of the upper Isaacs equation. An identical statement holds true for the lower value and the lower Isaacs equation. A version of the Dynamic Programming Principle is obtained as a by-product. If the Isaacs condition is satisfied, the game has a value over elementary (pure) strategies.
\end{abstract}

Keywords: Stochastic Perron's method, stochastic games, elementary strategies, viscosity solutions Mathematics Subject Classification (2010): 91A05, 91A15, 49L20, 49L25

\section{Introduction}

Continuous time games have been an active research area since the pioneering work of Isaacs [sa65. In EK72, Elliott and Kalton studied a deterministic differential game, where one player (using strategies) observes continuously the actions of the other players, who plays open loop controls. This formulation of the game has been considered for the first time in stochastic framework in [FS89]. The viscosity solution approach previously used in deterministic setting turns out to be quite involved here, and, actually, one cannot prove the dynamic programming principle working directly with the value functions (see [FS89] or [Kat95]).

A different approach, resembling verification for classical solutions but in the context of non-smooth viscosity solutions, was employed in [Świ96a] and Świ96b] (first for deterministic problems and then for zero-sum stochastic games in Elliott-Kalton formulation). There, it was proven (using inf/sup convolution regularization of viscosity solutions in the spirit of [LS85] and an approximation of the state equation by non-degenerate diffusions) that viscosity sub/super-solutions satisfy the corresponding half of the DPP (so called sub/super-optimality conditions), therefore comparing to the value function. The unique continuous viscosity solution (obtained separately by PDE methods, most likely by Perron's method for viscosity solutions introduced in [Ish87]) is equal to the value function(s) and

\footnotetext{
${ }^{*}$ University of Texas at Austin, Department of Mathematics, 1 University Station C1200, Austin, TX, 78712. E-mail address: sirbu@math.utexas.edu. The research of this author was supported in part by the National Science Foundation under Grant DMS 1211988. Any opinions, findings, and conclusions or recommendations expressed in this material are those of the authors and do not necessarily reflect the views of the National Science Foundation.
} 
the DPP is satisfied. In order to regularize the diffusion, the probability space needs to be enlarged to accommodate an independent Brownian Motion. This means that verification is actually performed for a slightly different game, not the one in the original formulation. Similar technical arguments to prove DPP for games in Elliott-Kalton formulation were used very recently in Kry13a and Kry13b for non-degenerate diffusions, but with only measurable in space coefficients.

Out of the existing literature on games, most papers (all above) use the Elliott-Kalton formulation previously mentioned, where one player uses controls and the other uses strategies. This asymmetric formulation produces two values of the game that do not compare by definition, as one would expect. It seems more natural to consider a symmetric formulation of the game. One way to do this, is to consider, for both players, strategies which are based only on the knowledge of the past of the state. Each player's actions are responses to the other player's actions through the use of such feed-back strategies. Feed-back strategies have already been used in both one-player (control) problems (see the notion of natural controls in Kry09) and in (discretized) zero-sum deterministic games (under the name of positional/historical strategies, and used by one or both players) in [KS88. From the recent literature on stochastic games, CR09. consider a game in the strong formulation over feed-back strategies with delay, but even allow for some mixing, and [PZ12] use discretized feed-back strategies (under the name of feed-back controls borrowed from the more classical control literature). In the weak formulation of a non-Markovian game over feed-back strategies, [PZ12] use BSDE's techniques to conclude that the values of the games are solutions to the path-dependent Isaacs equations. This work follows a long line of interesting results relating BSDEs to stochastic games in Elliott-Kalton formulation: HL95, [EKH03] or BL08, to name just a few.

We provide here a novel tool to study continuous-time zero-sum stochastic differential games, in an (arguably) more natural formulation. The aim of the current paper is two-fold:

1. to consider a symmetric formulation of the stochastic game over a (restricted class of) feed-back strategies that yield strong solutions to the state equations. The restriction is quite different, and (we believe) more natural than the delay in [CR09] or the deterministic time-discretization in [KS88] and [PZ12]. We find it conceptually important to have a class of strategies where the state is a one-to-one response to the (unobserved) noise, once both players have decided on what strategies will be used,

2. to show that the Stochastic Perron Method (introduced in BS12 for linear problems, later for Dynkin games in [BS14] and for control problems in [BS13]) can be adapted to differential games in the above formulation. This amounts to a new method to study games, and actually turns out to be rather elementary compared to the existing work.

In order to illustrate our approach, we impose some restrictive conditions on the controlled stochastic system, rather than consider the most general framework. More precisely: the state space is the whole $\mathbb{R}^{d}$, the state equation is uniformly locally Lipschitz in the state variable, the terminal pay-off is bounded, the controls live in some compact spaces, etc. It is well known from [FS89 that stochastic games are non-trivial even with (more) restrictive assumptions, as the strategic nature of the problem remains intact. We also consider only a terminal pay-off, but running cost can be easily added. However, we allow for the state equation to be degenerate, so one cannot expect any smoothness of solutions to the Bellman-Isaacs equation(s).

The method we present here amounts to a verification result for non-smooth viscosity solutions. The Stochastic Perron construction and viscosity comparison show that the value function(s) is the unique viscosity solution of the Bellman-Isaacs equation(s) and that (a version of) the DPP holds. Aside from the symmetric definition of the game over feed-back strategies, our verification program is conceptually different from the verification arguments in [Świ96a], [Świ96b. We work directly with the state equation and the original definition of the game. Our approach does not approximate the state equation by more regular diffusions so we have a verification argument for the original (strong) definition of the game on the initially chosen physical space that does not need to accommodate 
an additional independent Brownian motion. Technically, the Stochastic Perron method is rather elementary and is based only on how we define the stochastic semi-solutions, the well known definition of viscosity solutions and Itô's Lemma. Our verification is completed by a comparison argument that has to be proved analytically and separately anyway (here and in other existing approaches).

\section{Stochastic Differential Games and Strategies}

\subsection{The Stochastic System}

We consider a stochastic differential game with two players. The first player's actions belong to a compact metric space $\left(U, d_{u}\right)$ (usually $\left.U \subset \mathbb{R}^{k}\right)$. The second player's actions belong also to a compact metric space $\left(V, d_{v}\right)$ (usually $V \subset \mathbb{R}^{l}$ ). We assume that the state lives in the whole space $\mathbb{R}^{d}$ (but one could also consider a natural open domain $\mathcal{O} \subset \mathbb{R}^{d}$ as in [BS13]). Let $b:[0, T] \times \mathbb{R}^{d} \times U \times V \rightarrow \mathbb{R}^{d}$ and $\sigma:[0, T] \times \mathbb{R}^{d} \times U \times V \rightarrow \mathbb{M}_{d, d^{\prime}}$ be two continuous functions. We consider the diffusion

$$
\left\{\begin{array}{l}
d X_{t}=b\left(t, X_{t}, u_{t}, v_{t}\right) d t+\sigma\left(t, X_{t}, u_{t}, v_{t}\right) d W_{t} \\
X_{s}=x \in \mathbb{R}^{d}
\end{array}\right.
$$

starting at an initial time $s$ at some position $x$, and which is controlled by both players. Here, $W$ is a $d^{\prime}$-dimensional Brownian motion on a fixed probability space $(\Omega, \mathcal{F}, \mathbb{P})$. We intend to pose the problem in such a way that the state equation will admit strong solutions. Therefore, is is natural to assume that, for the initial time $s$, the filtration considered is $\mathbb{F}^{s}=\left(\mathcal{F}_{t}^{s}\right)_{s \leq t \leq T}$, the augmented natural filtration generated by the Brownian increments starting at $s$, by which we mean.

$$
\mathcal{F}_{t}^{s}=\sigma\left(W_{u}-W_{s}, s \leq u \leq t\right) \vee \mathcal{N}(\mathbb{P}, \mathcal{F}) \text { for } s \leq t \leq T .
$$

However, any larger filtration that keeps $W .-W_{s}$ a Brownian motion starting at $\mathrm{s}$ and satisfying the usual conditions is also fine. Now, given a bounded and continuous function $g: \mathbb{R}^{d} \rightarrow \mathbb{R}$, the second player pays to the first player the amount $\mathbb{E}\left[g\left(X_{T}^{s, x ; u, v}\right)\right]$. This leads to a zero-sum game of the form

$$
\sup _{u} \inf _{v} \mathbb{E}\left[g\left(X_{T}^{s, x ; u, v}\right)\right], \quad \inf _{v} \sup _{u} \mathbb{E}\left[g\left(X_{T}^{s, x ; u, v}\right)\right] .
$$

Just for the sake of presentation, we have assumed that no running cost is present, as in BS13. Such cost can easily be added. The lower and the upper Hamiltonians are defined by

$$
\begin{aligned}
& H^{-}(t, x, p, M):=\sup _{u \in U} \inf _{v \in V}\left[b(t, x, u, v) \cdot p+\frac{1}{2} \operatorname{Tr}\left(\sigma(t, x, u, v) \sigma(t, x, u, v)^{T} M\right)\right], \quad 0 \leq t \leq T, x \in \mathbb{R}^{d}, \\
& H^{+}(t, x, p, M):=\inf _{v \in V} \sup _{u \in U}\left[b(t, x, u, v) \cdot p+\frac{1}{2} \operatorname{Tr}\left(\sigma(t, x, u, v) \sigma(t, x, u, v)^{T} M\right)\right], \quad 0 \leq t \leq T, x \in \mathbb{R}^{d} .
\end{aligned}
$$

We associate, formally for the moment, the following Isaacs equations to the game

$$
\left\{\begin{array}{l}
-v_{t}-H^{i}\left(t, x, v_{x}, v_{x x}\right)=0 \text { on }[0, T) \times \mathbb{R}^{d} \\
v(T, \cdot)=g(\cdot), \text { on } \mathbb{R}^{d}
\end{array}\right.
$$

For $i=-$ we have the lower Isaacs equation, and for $i=+$ we have the upper Isaacs equation. We make the standing assumption that that the coefficients $b, \sigma$ of the stochastic system are jointly continuous, satisfy a uniform local Lipschitz condition in $x$, i.e.

$$
\text { (L) }|b(t, x, u, v)-b(t, y, u, v)|+|\sigma(t, x, u, v)-\sigma(t, y, u, v)| \leq L(K)|x-y|
$$


$\forall|x|,|y| \leq K, t \in[0, T], u \in U, v \in V$ for some $L(K)<\infty$, and also satisfy a global linear growth condition in $x$

$$
(\mathbf{L G}) \quad|b(t, x, u, v)|+|\sigma(t, x, u, v)| \leq C(1+|x|)
$$

$\forall|x|,|y| \in \mathbb{R}^{d}, t \in[0, T], u \in U, v \in V$ for some $C<\infty$. Under these assumptions, if both players choose to hold a constant strategy, the controlled state has a unique solution. This holds true even if the game is started at a random (but stopping) time sequel to the starting time $s$, and the constant strategies depend on whatever happened before the starting time. More precisely, we have the following proposition:

Proposition 2.1 Let $s \leq \tau^{\prime} \leq T$ be a stopping time with respect to the filtration $\mathbb{F}^{s}=\left(\mathcal{F}_{t}^{s}\right)_{s \leq t \leq T}$. Let $\xi \in \mathbb{R}^{d}, a \in U, b \in V$, be random variables measurable with respect to $\mathcal{F}_{\tau^{\prime}}^{s}$. Then, the system starting at time $\tau^{\prime}$ with initial condition $\xi$, where both players choose constant strategies $a, b$ in between $\tau^{\prime}$ and $T$ has a (path-wise) unique strong solution. In other words, the SDE

$$
\left\{\begin{array}{l}
d X_{t}=b\left(t, X_{t}, a, b\right) d t+\sigma\left(t, X_{t}, a, b\right) d W_{t}, \tau^{\prime} \leq t \leq T \\
X_{\tau^{\prime}}=\xi
\end{array}\right.
$$

has a unique strong solution $\left(X_{t}\right)_{\tau^{\prime} \leq t \leq T}$. If $\xi$ is square integrable, the solution is square integrable as well.

Proof: the arguments are standard, so we omit the details. The path-wise (or strong) uniqueness is proved by localization and Gronwall. Using again localization and then a Picard iteration, we prove the existence of a saturated strong solution, up to some explosion time. The global growth condition and Gronwall inequality insure that there is actually no explosion. $\diamond$

\subsection{Elementary Strategies}

So far, the game is defined formally. To have a consistent mathematical definition, we need to define some non-constant strategies for each player. In our model both players observe continuously the state process (which is a response to both players actions) but not the noise, and both players play (symmetrically) strategies based on the information available. This leads to a formulation of the game where the upper and the lower values compare by definition, as in in CR09] and, more recently, in PZ12. In other words, we propose for use as strategies, by both players:

1. functionals of the past of the state (pure feed-back strategies), if one expects the game to have a value. This is the case in [CR09] (with delay) and [PZ12] (for the weak formulation), following the older work [KS88] on deterministic games, where feed-back strategies were discretized with respect to time,

2. strategies as above, but mixed (at each time), if the game over pure strategies as above does not have a value. Some mixing is actually allowed in [CR09] and is also used in [KS88] for the case of deterministic games.

In other words, we believe that the game should be first formulated over such pure (feed-back) strategies and, if the lower and upper values are not equal, then one should proceed directly to considering mixed strategies where the game is expected to have a value. For mathematical reasons, we treat in the present paper the complete picture of the game over pure strategies. More precisely, we study separately the two value functions as solutions of the two Isaacs equations. If the Isaacs condition is satisfied, then, obviously, the game is expected to have a value over pure strategies. We defer the study of elementary mixed strategies in the absence of Isaacs condition to forthcoming work [Sî3.

We do not assume any delay as in CR09 for two reasons: such strategies don't work well with Stochastic Perron's Method and, conceptually, we find it harder to justify delaying the information available, while, at the same time, continuously changing actions. Some other natural restriction 
has still to be imposed on the feed-back strategies, so that, once each player chooses a strategy, the state equation has a unique strong solution. Despite the fact the noise is unobserved, we believe it is conceptually important for the state equation to have a unique strong solution over pure strategies. In addition, our so called Elementary Strategies that we propose below, are exactly the kind of strategies that one can expect to enforce in a continuous-time game, where changing actions continuously may not be completely feasible, but when a change is made, the information available should not be delayed and only used later. The Elementary Strategies are defined in a very similar manner to how one defines Elementary Integrands in stochastic integration, but on the state path space.

To simplify notation, for the remainder of the paper, fixed a starting time $s$, we denote by $C([s, T]) \triangleq C\left([s, T], \mathbb{R}^{d}\right)$ and endow this path space with the natural (and raw) filtration $\mathbb{B}^{s}=$ $\left(\mathcal{B}_{t}^{s}\right)_{s \leq t \leq T}$ defined by

$$
\mathcal{B}_{t}^{s} \triangleq \sigma(y(u), s \leq u \leq t), \quad s \leq t \leq T .
$$

The elements of the path space $C([s, T])$ will be, usually, denoted by $y(\cdot)$ or $y$, when there is no risk of confusion. The stopping times on the space $C([s, T])$ with respect with the filtration $\mathbb{B}^{s}$, i.e. mappings $\tau: C([s, T]) \rightarrow[s, T]$ satisfying $\{\tau \leq t\} \in \mathcal{B}_{t}^{s} \forall s \leq t \leq T$ are called stopping rules, following [KS01]. We denote by $\mathbb{B}^{s}$ the class of such stopping rules starting at $s$.

Definition 2.2 (Elementary Pure Strategies) Fix $0 \leq s \leq T$. An elementary strategy u starting at $s$, for the first player, is defined by

- a finite non-decreasing sequence of stopping rules, i.e. $\tau_{k} \in \mathbb{B}^{s}$ for $k=1, \ldots, n$ and

$$
s=\tau_{0} \leq \ldots \tau_{k} \leq \cdots \leq \tau_{n}=T
$$

- for each $k=1 \ldots n$, a constant value of the strategy $\xi_{k}$ in between the times $\tau_{k-1}$ and $\tau_{k}$, which is decided based only on the knowledge of the past state up to $\tau_{k-1}$, i.e. $\xi_{k}: C([s, T]) \rightarrow U$ such that $\xi_{k} \in \mathcal{B}_{\tau_{k-1}}^{s}$.

The strategy is to hold $\xi_{k}$ in between $\left(\tau_{k-1}, \tau_{k}\right]$, i.e. $u:(s, T] \times C([s, T]) \rightarrow U$ is defined by

$$
u(t, y(\cdot)) \triangleq \sum_{k=1}^{n} \xi_{k}(y(\cdot)) 1_{\left\{\tau_{k-1}(y(\cdot))<t \leq \tau_{k}(y(\cdot))\right\}} \cdot
$$

Such a strategy can be denoted, when there is no risk of confusion, also as $u=\left(\xi_{k}, \tau_{k}\right)_{k=1, n}$. An elementary strategy $v$ for the second player is defined in an identical way, but takes values in $V$. We denote by $\mathcal{U}(s, s)$ and $\mathcal{V}(s, s)$ the collections of all possible elementary strategies for the first, and the second player, given the initial deterministic time $s$.

The timings $\tau_{k}$ of changing the controls in the definition of strategies, are exactly the "stopping rules" considered in the game of stopping and control in [KS01. In addition, we would like to point out that, since $\xi_{1} \in \mathcal{B}_{s}^{s}$, this means that $\xi_{1}(\cdot)=l(y(s))$, for some measurable function $l: \mathbb{R}^{d} \rightarrow U$. It is obvious that, for a simple strategy, the representation $u=\left(\xi_{k}, \tau_{k}\right)_{k=1, n}$ is not unique.

Remark 2.3 The elementary strategies are obviously non-anticipative functionals of the paths of $X$ but they are neither

1. Lipschitz with respect to the sup norm on the path-space (this allows for great flexibility in pursuing Stochastic Perron)

2. nor delayed strategies as considered in [CR09]

Lipschitz functionals of the path are good strategies to define the values of the game (resulting in strong solutions of the state equation, with the very strong additional Lipschitz assumption of coefficients) but hard to fit in the framework of Stochastic Perron we develop here. In addition, we believe that our "discrete-time" strategies are easier to justify in applications. 
Fortunately, the strategies we just defined still produce strong solutions of the state equation without any Lipschitz assumption in $u, v$. More precisely, iterating Proposition 2.1, we get

Proposition 2.4 Fix $s, x$ and let players one and two choose strategies $u \in \mathcal{U}(s, s)$ and $v \in \mathcal{V}(s, s)$. Then, there exists a unique strong (and square integrable) solution $\left(X_{t}^{s, x ; u, v}\right)_{s \leq t \leq T}$ of the state equation

$$
\left\{\begin{array}{l}
d X_{t}=b\left(t, X_{t}, u(t, X .), v(t, X .) d t+\sigma\left(t, X_{t}, u(t, X .), v(t, X .)\right) d W_{t}, s \leq t \leq T\right. \\
X_{s}=x \in \mathbb{R}^{d} .
\end{array}\right.
$$

Uniqueness holds in the path-wise sense.

The proof of the above proposition is based on iterating Proposition 2.1 together with the following very simple but useful lemma:

Lemma 2.5 Fix $s$ and let $\tau$ be a stopping rule, $\tau: C([s, T]) \rightarrow[s, T], \tau \in \mathbb{B}^{s}$. Let $\left(X_{t}\right)_{s \leq t \leq T}$ be a process with continuous (all, not only almost surely) paths, which is adapted to $\mathbb{F}^{s}$. Then, the random time $\tau_{X}: \Omega \rightarrow[s, T]$ defined by $\tau_{X}(\omega) \triangleq \tau(X .(\omega))$ is a stopping time w.r.to the filtration $\mathbb{F}^{s}$. In addition $X_{\tau_{X}} \in \mathcal{F}_{\tau_{X}}^{s}$.

Remark 2.6 In one-player (control) problems, the use of open-loop controls yields strong solutions of the state equation (because of the Lipschitz condition in the standing assumptions). Something similar would happen if one defined the game over open-loop controls, but this formulation is not appropriate for games. In the Elliott-Kalton formulation of [FS89], where one player chooses open-loop controls and the other strategies, again, existence of strong solutions of the state equation is easily checked. Once feed-back strategies are allowed (actually for both players), the existence of strong solutions is non-trivial. One can, therefore, either restrict strategies, as in [CR09] in order to have a strong formulation, or consider a weak formulation as in [PZ12] (but restriction are needed even in the weak formulation). We choose here the strong formulation, and restrict the strategies to the class of elementary strategies. The restriction to the class of elementary strategies is meaningful both at the modeling level (actions can only be changed discretely in time) and at the level of the mathematics (we need the state equation to be well posed).

We now formulate the game rigorously. For fixed $0 \leq s \leq t \leq T$ and $x \in \mathbb{R}^{d}$ (deterministic), we define the lower and the upper value of the game

$$
V^{-}(s, x) \triangleq \sup _{u \in \mathcal{U}(s, s)} \inf _{v \in \mathcal{V}(s, s)} \mathbb{E}\left[g\left(X_{T}^{s, x: u, v}\right)\right] \leq \inf _{v \in \mathcal{V}(s, s)} \sup _{u \in \mathcal{U}(s, s)} \mathbb{E}\left[g\left(X_{T}^{s, x ; u, v}\right)\right] \triangleq V^{+}(s, x) .
$$

Since both our players play strategies (symmetrically), the lower and the upper values compare by definition. This feature has already been emphasized in the literature, like [CR09] or [PZ12, in opposition to the Elliott-Kalton formulation in [FS89]. A finite valued version of feed-back strategies is present in PZ12 for technical reasons, but the discretization is over deterministic times, and the game is considered in weak formulation. Our strategies are actually not finitely valued: they are discrete only in time. The discretization over deterministic times in PZ12] is also reminiscent of the classic work [Nis88, where the author uses a fixed time grid and then passes to the limit using semi-group techniques.

The lower value of the game has the meaning that player 1 announces his/her full strategy to player 2 , who chooses a strategy accordingly. Symmetrically for the upper value. Of course, something like this does not really happen, so, if the two values differ, one should just consider mixed strategies. However, we do analyze the two possibly different values for mathematical reasons. Assume for a moment, that, player 2, indeed, knows the full strategy that player 1 is going to use. If that is the case, since both players' strategies are non-anticipative functionals of the path, conceptually, this would correspond exactly to the Elliott-Kalton idea that player 2 observes continuously player's 1 controls. It is, therefore, not surprising that we expect such lower value and upper value, to be the 
solutions to the corresponding Isaacs equation. However, such a heuristic statement needs a proof, and part of our contribution is to provide such proof.

We also need need to define strategies starting at sequel times to the initial (deterministic) time $s$. The starting time is a stopping rule.

Definition 2.7 (Elementary Strategies starting later) Fix $s$ and let $\tau \in \mathbb{B}^{s}$ be a stopping rule. An elementary strategy, denoted by $u \in \mathcal{U}(s, \tau)$, for the first player, starting at $\tau$, is defined by

- (again) a finite non-decreasing sequence of stopping rules, i.e. $\tau_{k} \in \mathbb{B}^{s}, k=1, \ldots n$ for some finite $n$, and with $\tau=\tau_{0} \leq \ldots \tau_{k} \leq \cdots \leq \tau_{n}=T$.

- for each $k=1 \ldots n$, a constant action $\xi_{k}$ in between the times $\tau_{k-1}$ and $\tau_{k}$, which is decided based only on the knowledge of the past state up $\tau_{k-1}$, i.e. $\xi_{k}: C([s, T]) \rightarrow U$ such that $\xi_{k} \in \mathcal{B}_{\tau_{k-1}}$.

The strategy is, again, to hold $\xi_{k}$ in between $\left(\tau_{k-1}, \tau_{k}\right]$, i.e..

$$
u:\{(t, y) \mid \tau(y)<t \leq T, y \in C([s, T])\} \rightarrow U \quad \text { with } \quad u(t, y(\cdot)) \triangleq \sum_{k=1}^{n} \xi_{k}(y(\cdot)) 1_{\left\{\tau_{k-1}(y(\cdot))<t \leq \tau_{k}(y(\cdot))\right\}} .
$$

We define similarly $\mathcal{V}(s, \tau)$ for the second player.

It is clear that, if we consider $\tau=s$ in the Definition 2.7 of $\mathcal{U}(s, \tau)$, we obtain the same thing as $\mathcal{U}(s, s)$ in Definition 2.2, so the notation is consistent. The same holds true for $\mathcal{V}$. The classes of strategies $\mathcal{U}(s, \tau)$ and $\mathcal{V}(s, \tau)$ are, obviously, not closed in any reasonable sense, but they are robust. The two results below have very simple proofs, which we omit.

Lemma 2.8 Let $u_{1}, u_{2} \in \mathcal{U}(s, \tau)$ for some $s \leq T$ and $\tau \in \mathbb{B}^{s}$. Let $A \subset C\left([s, T]\right.$ such that $A \in \mathbb{B}_{\tau}^{s}$. Then, the mapping $u:(s, T] \times C([s, T]) \rightarrow U$ defined by

$$
u(t, y)=1_{\{y \in A\}} u_{1}(t, y)+1_{\left\{y \in A^{c}\right\}} u_{2}(t, y),
$$

is an elementary strategy in $\mathcal{U}(s, \tau)$. An identical statement holds for the second player.

Strategies in $\mathcal{U}(s, \tau)$ or $\mathcal{V}(s, \tau)$ cannot be used by themselves for the game starting at $s$, but have to be concatenated with other strategies.

Proposition 2.9 (Concatenated elementary strategies) Fix $s$ and let $\tau \in \mathbb{B}^{s}$ be a stopping rule and $\tilde{u} \in \mathcal{U}(s, \tau)$. Then, for each $u \in \mathcal{U}(s, s)$, the mapping $u \otimes_{\tau} \tilde{u}:(s, T] \times C([s, T]) \rightarrow U$ defined by

$$
\left(u \otimes_{\tau} \tilde{u}\right)(t, y(\cdot)) \triangleq u(t, y(\cdot)) 1_{\{s<t \leq \tau(y(\cdot))\}}+\tilde{u}(t, y(\cdot)) 1_{\{\tau(y(\cdot))<t \leq T\}}
$$

is a simple strategy starting at $s$, i.e. $u \otimes_{\tau} \tilde{u} \in \mathcal{U}(s, s)$. A similar statement holds for the second player.

\section{Stochastic Perron's Method}

Having defined elementary strategies, and the concatenation, we are now in position to define stochastic semi-solutions of the two Isaacs equations.

Definition 3.1 (Stochastic Super-Solution of Upper Isaacs) A function $w:[0, T] \times \mathbb{R}^{d} \rightarrow \mathbb{R}$ is called a stochastic super-solution of the upper Isaacs equation if

1. it is bounded, continuous and $w(T, \cdot) \geq g(\cdot)$, 
2. for each $s$ and for each stopping rule $\tau: C([s, T]) \rightarrow[s, T], \tau \in \mathbb{B}^{s}$ there exists an elementary strategy $\tilde{v} \in \mathcal{V}(s, \tau)$ such that, for any $u \in \mathcal{U}(s, s)$, any $v \in \mathcal{V}(s, s)$, any $x \in \mathbb{R}^{d}$ and each stopping rule $\rho \in \mathbb{B}^{s}, \tau \leq \rho \leq T$, with the simplifying notation $X \triangleq X^{s, x, u, v \otimes_{\tau} \tilde{v}}$ and (see Lemma 2.5) $\tau^{\prime} \triangleq \tau(X), \rho^{\prime} \triangleq \rho(X)$, we have

$$
w\left(\tau^{\prime}, X_{\tau^{\prime}}\right) \geq \mathbb{E}\left[w\left(\rho^{\prime}, X_{\rho^{\prime}}\right) \mid \mathcal{F}_{\tau^{\prime}}^{s}\right] \mathbb{P}-\text { a.s. }
$$

Choosing $\tau=s$, we can see that, if $w$ is a stochastic super-solution, there exists $\tilde{v} \in \mathcal{V}(s, s)$ such that

$$
w(s, x) \geq \mathbb{E}\left[w\left(\rho\left(X^{s, x, u, \tilde{v}}\right), X_{\rho\left(X^{s, x, u, \tilde{v}}\right)}^{s, x, u, \tilde{F}}\right) \mid \mathcal{F}_{s}^{s}\right], \mathbb{P}-a . s
$$

for all $u \in \mathcal{U}(s, s)$ and $\rho \in \mathbb{B}^{s}$. After taking the expectation, it is now obvious that, if $w$ is a stochastic super-solution, then we have the half DPP for $w$, i.e.

$$
w(s, x) \geq \inf _{v \in \mathcal{V}(s, s)} \sup _{u \in \mathcal{U}(s, s)} \mathbb{E}\left[w\left(\rho\left(X^{s, x, u, v}\right), X_{\rho\left(X^{s, x, u, v}\right)}^{s, x, u, v}\right)\right], \quad \forall \rho \in \mathbb{B}^{s} .
$$

Since $w(T, \cdot) \geq g(\cdot)$, we obtain easily that $w(s, x) \geq V^{+}(s, x)$. Compared to the Elliott-Kalton formulation prevalent in the literature, we have here a game where both players use elementary feed-back strategies. Also, in (5), stopping rules are considered instead of stopping times. Therefore, even if one tried to enlarge the probability space to accommodate an independent Brownian motion, it is not clear if the non-trivial approximation arguments in Śsi96b] could be reproduced to conclude that the super-optimality principle (5) is equivalent to the notion of viscosity super-solution of upper Isaacs equation. However, instead of following this direction, we circumvent most difficulties using the more elementary Stochastic Perron Method, which means taking the infimum of stochastic super-solutions.

The stochastic sub-solutions of the lower Isaacs equation are defined symmetrically, in an obvious way.

Definition 3.2 (Stochastic Sub-Solution of Lower Isaacs) A function $w:[0, T] \times \mathbb{R}^{d} \rightarrow \mathbb{R}$ is called a stochastic sub-solution of the lower Isaacs equation if

1. it is bounded, continuous and $w(T, \cdot) \leq g(\cdot)$,

2. for each $s$ and for each stopping rule $\tau \in \mathbb{B}^{s}$ there exists an elementary strategy $\tilde{u} \in \mathcal{U}(s, \tau)$ such that, for any $u \in \mathcal{U}(s, s)$, any $v \in \mathcal{V}(s, s)$, any $x$ and each stopping rule $\rho \in \mathbb{B}^{s}, \tau \leq \rho \leq T$, with the simplifying notation $X \triangleq X^{s, x, u \otimes_{\tau} \tilde{u}, v}$ and $\tau^{\prime} \triangleq \tau(X), \rho^{\prime} \triangleq \rho(X)$, we have

$$
w\left(\tau^{\prime}, X_{\tau^{\prime}}\right) \leq \mathbb{E}\left[w\left(\rho^{\prime}, X_{\rho^{\prime}}\right) \mid \mathcal{F}_{\tau^{\prime}}^{s}\right] \mathbb{P}-\text { a.s. }
$$

It is another easy observation that, a stochastic sub-solution of the lower Isaacs equation satisfies $w \leq V^{-}$and the corresponding half $\mathrm{DPP} /$ sub-optimality principle. The two definitions above are perfectly symmetric, and they would be enough to proceed with Stochastic Perron, in case the Isaacs condition holds. For the general case we study here we need to also define:

Definition 3.3 (Stochastic Sub-Solution of Upper Isaacs) A function $w:[0, T] \times \mathbb{R}^{d} \rightarrow \mathbb{R}$ is called a stochastic sub-solution of the upper Isaacs equation if

1. it is bounded, continuous and $w(T, \cdot) \leq g(\cdot)$,

2. for each $s$ and for each stopping rule $\tau: C\left([s, T] \rightarrow[s, T], \tau \in \mathbb{B}^{s}\right.$ and each strategy $v \in \mathcal{V}(s, s)$ there exists an elementary strategy $\tilde{u} \in \mathcal{U}(s, \tau)$ (depending on $v$ and $\tau$ ) such that, for any $u \in \mathcal{U}(s, s)$ and any $x$ as well as each stopping rule $\rho \in \mathbb{B}^{s}, \tau \leq \rho \leq T$, with the simplifying notation $X \triangleq X^{s, x, u \otimes_{\tau} \tilde{u}, v}$ and $\tau^{\prime} \triangleq \tau(X), \rho^{\prime} \triangleq \rho(X)$, we have

$$
w\left(\tau^{\prime}, X_{\tau^{\prime}}\right) \leq \mathbb{E}\left[w\left(\rho^{\prime}, X_{\rho^{\prime}}\right) \mid \mathcal{F}_{\tau^{\prime}}^{s}\right] \mathbb{P}-\text { a.s. }
$$


We omit the definition of Stochastic super-solution of lower Isaacs for the reason of being obvious by now. Let $w$ a stochastic sub-solution of upper Isaacs. Fix $v \in \mathcal{V}(s, s)$ and $\tau=s$. There exists $\tilde{U} \in \mathcal{U}(s, s)$ (depending on $v$ ) such that, for each $\rho \in \mathbb{B}^{s}$ we have

$$
w(s, x) \leq \mathbb{E}\left[w\left(\rho\left(X^{s, x, \tilde{u}, v}\right), X_{\rho\left(X^{s, x, u}, v\right)}^{s, x, \tilde{u}}\right) \mid \mathcal{F}_{s}^{s}\right], \mathbb{P}-a . s
$$

After taking the expectation, it is now obvious that, if $w$ is a stochastic super-solution, then we have the half DPP/sub-optimality principle for $w$, i.e.

$$
w(s, x) \leq \inf _{v \in \mathcal{V}(s, s)} \sup _{u \in \mathcal{U}(s, s)} \mathbb{E}\left[w\left(\rho\left(X^{s, x, u, v}\right), X_{\rho\left(X^{s, x, u, v}\right)}^{s, x, u, v}\right)\right], \quad \forall \rho \in \mathbb{B}^{s}
$$

Since $w(T, \cdot) \leq g(\cdot)$, we obtain easily that $w(s, x) \leq V^{+}(s, x)$.

Remark 3.4 The definitions of stochastic semi-solutions are quite different from those in the case of one player (control problems) in [BS13]. There, one simply starts the problem at a stopping time sequel to $s$ in the definition(s): the same does not make sense here. Therefore, the nearly optimal strategy (maybe depending on the other player strategy, as for the sub-solution of upper Isaacs) in the definition(s) has to be concatenated with any other strategy up to that stopping time rule $\tau$. This makes sense conceptually as one cannot simply start the game at $\tau$. On the other hand, if we tried to start the game at a stopping time $\tau^{\prime}$ of the original filtration $\mathbb{F}^{s}$, we would get into significant difficulty trying to complete Stochastic Perron's method below. In some sense, this is one of the best features of Stochastic Perron's Method, as it allows for great flexibility as to what we can choose for stochastic semi-solutions, as long as the definitions provide an easy comparison to the value function(s).

We denote by

1. $\mathcal{U}^{+}$the set of stochastic super-solutions of upper Isaacs

2. $\mathcal{U}^{-}$the set of stochastic sub-solutions of upper Isaacs

3. $\mathcal{L}^{+}$the set of stochastic super-solutions of lower Isaacs (not defined explicitly)

4. $\mathcal{L}^{-}$the set of stochastic sub-solutions of lower Isaacs.

Since $g$ is bounded, all the sets are obviously non-empty. As expected, by Stochastic Perron's Method we mean taking the sup of sub-solutions and/or inf of super-solutions (by which we mean stochastic semi-solutions):

$$
v^{-} \triangleq \sup _{w \in \mathcal{U}^{-}} w \leq V^{+} \leq \inf _{w \in \mathcal{U}^{+}} w \triangleq v^{+}
$$

and

$$
w^{-} \triangleq \sup _{w \in \mathcal{L}^{-}} w \leq V^{-} \leq \inf _{w \in \mathcal{L}^{+}} w \triangleq w^{+} .
$$

We can say, without need for any more proof, that $w^{-} \leq V^{-} \leq V^{+} \leq v^{+}$. We recall the standing assumptions: $g$ is continuous and bounded, $b$ and $\sigma$ are continuous on their whole corresponding domains, locally uniformly Lipschitz in $x$ and have global linear growth in $x$.

Theorem 3.5 (Stochastic Perron for games) Under the standing assumptions:

1. the function $v^{+}$is a bounded upper semi-continuous (USC) viscosity sub-solution of the upper Isaacs equation and satisfies the Half DPP (5). The function $v^{-}$is a bounded lower semicontinuous (LSC) viscosity super-solution of the upper Isaacs equation and satisfies the half $D P P(\underline{6})$.

2. the function $w^{+}$is a bounded upper semi-continuous (USC) viscosity sub-solution of the lower Isaacs equation, and the function $w^{-}$is a bounded lower semi-continuous (LSC) viscosity supersolution of the lower Isaacs equation (and they both satisfy the corresponding halves of the DPP for the lower equation). 
Remark 3.6 The viscosity semi-solution property holds at time $t=0$ as well, consistent with the notation in (2). This is important (as can be seen from Lemma 4.3) to obtain comparison up to time $t=0$ for semi-continuous viscosity solutions.

In order to prove the Theorem, we need two Lemmas.

Lemma 3.7 1. if $w_{1}, w_{2} \in \mathcal{U}^{+}$then $w_{1} \wedge w_{2} \in \mathcal{U}^{+}$

2. if $w_{1}, w_{2} \in \mathcal{U}^{-}$then $w_{1} \vee w_{2} \in \mathcal{U}^{-}$

Fix $\tau \in \mathbb{B}^{s}$ a stopping time strategy and. Let $\tilde{v}_{1}, \tilde{v}_{2} \in \mathcal{V}(s, \tau)$ be the two strategies, for the second player, starting at $\tau$ corresponding the the super-solutions $w_{1}$ and $w_{2}$. The new strategy starting at $\tau$ defined by

$$
\tilde{v}(t, y(\cdot))=\tilde{v}_{1}(t, y(\cdot)) 1_{\left\{w_{1}(\tau(y), y(\tau(y))) \leq w_{2}(\tau(y), y(\tau(y)))\right\}}+\tilde{v}_{2}(t, y(\cdot)) 1_{\left\{w_{1}(\tau(y), y(\tau(y)))>w_{2}(\tau(y), y(\tau(y)))\right\}}
$$

does the job for the definition of $w \triangleq w_{1} \wedge w_{2}$ as a stochastic super-solution of the upper Isaacs. A similar construction has to be made for the second part. $\diamond$

Lemma 3.8 There exists a non-increasing sequence $\mathcal{U}^{+} \ni w_{n} \searrow v^{+}$and an non-decreasing sequence $\mathcal{U}^{-} \ni v_{n} \nearrow v^{-}$.

Proof: according to Proposition 4.1 in BS12, there exist $\tilde{w}_{n} \in \mathcal{U}^{+}$such that $v^{+}=\inf _{n} \tilde{w}_{n}$. Now, we can just define $w_{n}=\tilde{w}_{1} \wedge \cdots \wedge \tilde{w}_{n} \in \mathcal{U}^{+} \searrow v^{+}$. A very similar proof works for the second part of the lemma. $\diamond$

Proof of Theorem 3.5. the proof of the half DPP is obvious, by construction, since we have seen that the stochastic semi-solution satisfy such half DPP. As far as the viscosity property is concerned, we only prove item 1 , as the second is obviously symmetric.

1. ( $v^{+}$is viscosity sub-solution of upper Isaacs)

1.1: The interior sub-solution property for $v^{+}$: Let $\left(t_{0}, x_{0}\right)$ in the parabolic interior $[0, T) \times \mathbb{R}^{d}$ such that a smooth function $\varphi$ strictly touches $v^{+}$from above at $\left(t_{0}, x_{0}\right)$. Assume, by contradiction, that

$$
\varphi_{t}+H^{+}\left(t, x, \varphi_{x}, \varphi_{x x}\right)<0 \text { at }\left(t_{0}, x_{0}\right) .
$$

In particular, there exists $\hat{v} \in V$ and $\varepsilon>0$ such that

$$
\varphi_{t}\left(t_{0}, x_{0}\right)+\sup _{u \in U}\left[b\left(t_{0}, x_{0}, u, \hat{v}\right) \cdot \varphi_{x}\left(t_{0}, x_{0}\right)+\frac{1}{2} \operatorname{Tr}\left(\sigma\left(t_{0}, x_{0}, u, \hat{v}\right) \sigma(t, x, u, \hat{v})^{T} \varphi_{x x}\left(t_{0}, x_{0}\right)\right)\right]<-\varepsilon .
$$

To simplify notation, all small balls here are actually included in (i.e. intersected with) the parabolic interior. Since $b, \sigma$ are continuous, and $U$ is compact, the uniform continuity of the above expression in $(t, x, u)$ for $(t, x)$ around $\left(t_{0}, x_{0}\right)$ implies that there exists a smaller $\varepsilon>0$ such that

$$
\varphi_{t}(t, x)+\sup _{u \in U}\left[b(t, x, u, \hat{v}) \cdot \varphi_{x}(t, x)+\frac{1}{2} \operatorname{Tr}\left(\sigma(t, x, u, \hat{v}) \sigma(t, x, u, \hat{v})^{T} \varphi_{x x}(t, x)\right)\right]<-\varepsilon, \quad \text { on } B\left(t_{0}, x_{0}, \varepsilon\right) .
$$

Now, on the compact (rectangular) torus $\mathbb{T}=\overline{B\left(t_{0}, x_{0}, \varepsilon\right)}-B\left(t_{0}, x_{0}, \varepsilon / 2\right)$ we have that $\varphi>v^{+}$and the min of $\varphi-v^{+}$is attained, therefore it is strictly positive. In other words $\varphi>v^{+}+\eta$ on $\mathbb{T}$ for some $\eta>0$. Since $w_{n} \searrow v^{+}$, a Dini type argument similar to BS14 and BS13] shows that, for $n$ large enough we have $\varphi>w_{n}+\eta / 2$. For simplicity, fix such an $n$ and call $w=w_{n}$. Now, define, for small $\delta<<\eta / 2$

$$
w^{\delta} \triangleq\left\{\begin{array}{l}
(\varphi-\delta) \wedge w \text { on } B\left(t_{0}, x_{0}, \varepsilon\right) \\
w \text { outside } B\left(t_{0}, x_{0}, \varepsilon\right)
\end{array}\right.
$$

Since $w^{\delta}\left(t_{0}, x_{0}\right)<v^{+}\left(t_{0}, x_{0}\right)$, we obtain a contradiction if we can show $w^{\delta} \in \mathcal{U}^{+}$. 
In order to do so, fix $s$ and let $\tau \in \mathbb{B}^{s}$ be a stopping rule for the initial time $s$. We need to construct an elementary strategy $\tilde{v} \in \mathcal{V}(s, \tau)$ in the Definition of stochastic super-solution for $w^{\delta}$. This can be done as follows: since $w$ is a stochastic super-solution of upper Isaacs, there exists an elementary strategy $\tilde{v}_{1}$ for $w$ starting at $\tau$ that does the job. Then

1. if $(\varphi-\delta)<w$ at $\tau$, follow the constant action $\hat{v}$.

2. if $(\varphi-\delta) \geq w$ at $(\tau, \xi)$ follow the strategy $\tilde{v}_{1}$

3. follow the strategy defined in 1-2 until the first time $\tau_{1}$ when $\left(t, X_{t}\right) \in \partial B\left(t_{0}, x_{0}, \varepsilon / 2\right)$. On this boundary, we know that $w^{\delta}=w$.

4. after this, follow the strategy $\tilde{v}_{3} \in \mathcal{V}\left(s, \tau_{1}\right)$ corresponding to the stochastic sub-solution $w$ with starting stopping rule $\tau_{1}$

In a rigorous notation though, we first define $\tilde{v}_{2} \in \mathcal{V}(s, \tau)$ by

$$
\tilde{v}_{2}(t, y(\cdot))=\hat{v} 1_{\{\varphi(\tau(y), y(\tau(y)))-\delta<w(\tau(y), y(\tau(y)))\}}+\tilde{v}_{1}(t, y(\cdot)) 1_{\{\varphi(\tau(y), y(\tau(y)))-\delta \geq w(\tau(y), y(\tau(y)))\}} \cdot
$$

Denote now by $\tau_{1}: C([s, T]) \rightarrow[s, T], \tau \leq \tau_{1} \leq T$,

$$
\tau_{1}(y) \triangleq \inf _{\tau(y) \leq t \leq T}\left\{(t, y(t)) \in \partial B\left(t_{0}, x_{0}, \varepsilon / 2\right)\right\} .
$$

Now, if $\tilde{v}_{3} \in \mathcal{V}\left(s, \tau_{1}\right)$ is the strategy from the definition of the stochastic super-solution $w$, corresponding to stopping rule $\tau_{1}$, then we can define

$$
\tilde{v}=\tilde{v}_{2} \otimes_{\tau_{1}} \tilde{v}_{3} \in \mathcal{V}(s, \tau)
$$

to finish the proof. In order to check that $\tilde{v}$ fulfills the condition in the definition of stochastic supersolution for upper Isaacs, one has to follow similar arguments to the proofs in [BS13], based on Itô's Lemma applied to $d(\varphi-\delta)\left(t, X_{t}\right)$, conditioning and the tower property. However, one has to shift attention from stopping times as in BS13 to stopping rules. We present the arguments in what follows. Fix $u \in \mathcal{U}(s, s), v \in \mathcal{V}(s, s), x \in \mathbb{R}^{d}$ and choose a stopping rule $\rho \in \mathbb{B}^{s}$ with $\tau \leq \rho \leq T$. Denote by $X \triangleq X^{s, x, u, v \otimes_{\tau} \tilde{v}}$, where $\tilde{v}$ was just defined above and $\tau^{\prime} \triangleq \tau(X), \rho^{\prime} \triangleq \rho(X)$. Let also $\tau_{1}^{\prime} \triangleq \tau_{1}(X)$ such that $\tau^{\prime} \leq \tau_{1}^{\prime} \leq \rho^{\prime}$, and define the event $A \triangleq\left\{(\varphi-\delta)\left(\tau^{\prime}, X_{\tau^{\prime}}\right)<w\left(\tau^{\prime}, X_{\tau^{\prime}}\right)\right\}$. We first observe that

$$
X_{t}=1_{A} X_{t}^{s, x, u, v \otimes_{\tau} \hat{v}}+1_{A^{c}} X_{t}^{s, x, u, v \otimes_{\tau} \tilde{v}_{1}}, \tau^{\prime} \leq t \leq \tau_{1}^{\prime} .
$$

On $A$, the process $(\varphi-\delta)\left(\cdot, X\right.$.) then satisfies the super-martingale property in between $\tau^{\prime}$ and $\tau_{1}^{\prime}$ from Itô's Lemma. On $A^{c}$, the process $w\left(\cdot, X\right.$.) satisfies the super-martingale inequality in between $\tau^{\prime}$ and $\tau_{1}^{\prime}$ from the definition of the strategy $\tilde{v}_{1} \in \mathcal{V}(s, \tau)$. Putting this together, we have

$$
w^{\delta}\left(\tau^{\prime}, X_{\tau^{\prime}}\right) \geq \mathbb{E}\left[w^{\delta}\left(\tau_{1}^{\prime}, X_{\tau_{1}^{\prime}}\right) \mid \mathcal{F}_{\tau^{\prime}}^{s}\right] \mathbb{P}-\text { a.s. }
$$

Now, using the fact that $\tilde{v}_{3} \in \mathcal{V}\left(s, \tau_{1}\right)$ provides a super-martingale inequality concatenated with any previous strategy $v$ and against any strategy $u$ of the opponent, we have

$$
w^{\delta}\left(\tau_{1}^{\prime}, X_{\tau_{1}^{\prime}}\right)=w\left(\tau_{1}^{\prime}, X_{\tau_{1}^{\prime}}\right) \geq \mathbb{E}\left[w\left(\rho^{\prime}, X_{\rho^{\prime}}\right) \mid \mathcal{F}_{\tau_{1}^{\prime}}^{s}\right] \geq \mathbb{E}\left[w^{\delta}\left(\rho^{\prime}, X_{\rho^{\prime}}\right) \mid \mathcal{F}_{\tau_{1}^{\prime}}^{s}\right] \mathbb{P}-\text { a.s. }
$$

The iterating conditioning provides $w^{\delta}\left(\tau^{\prime}, X_{\tau^{\prime}}\right) \geq \mathbb{E}\left[w^{\delta}\left(\rho^{\prime}, X_{\rho^{\prime}}\right) \mid \mathcal{F}_{\tau^{\prime}}^{s}\right] \mathbb{P}-$ a.s. This means $w^{\delta} \in \mathcal{U}^{+}$, so we reached a contradiction.

1.2. The terminal condition property for $v^{+}$: the proof is quite similar to the proof of 1.1. More precisely, first we argue by contradiction, similar to either above or to BS13. (but easier since controls are compact), then we construct a strategy $\tilde{v}$ as above. Finally, we follow the arguments above based on Itô's Lemma and conditioning, to finish the proof. We develop these ideas below. 
Assume that, for some $x_{0} \in \mathbb{R}^{d}$ we have $v^{+}\left(T, x_{0}\right)>g\left(x_{0}\right)$. Since $g$ is USC, there exists an $\varepsilon>0$ such that $g(x) \leq v^{+}\left(T, x_{0}\right)-\varepsilon$ if $\left|(t, x)-\left(T, x_{0}\right)\right| \triangleq \max \left\{\left|x-x_{0}\right|, T-t\right\} \leq \varepsilon$. Denote, once again, by

$$
\left.\mathbb{T} \triangleq \overline{B\left(T, x_{0}, \varepsilon\right)}-B\left(T, x_{0}, \varepsilon / 2\right)\right) \cap\left([0, T] \times \mathbb{R}^{d}\right) .
$$

Now $v^{+}$is USC so bounded on $\mathbb{T}$. Choose $\eta>0$ small enough so that

$$
v^{+}\left(T, x_{0}\right)+\frac{\varepsilon^{2}}{4 \eta}>\varepsilon+\sup _{(t, x) \in \mathbb{T}} v^{+}(t, x) .
$$

Since the inequality above is strict, we use again a decreasing sequence and a Dini argument from BS14] to find some $w \in \mathcal{V}^{+}$such that

$$
v^{+}\left(T, x_{0}\right)+\frac{\varepsilon^{2}}{4 \eta}>\varepsilon+\sup _{(t, x) \in \mathbb{T}} w(t, x) .
$$

We now define, for $k>0$ the following function

$$
\varphi^{\eta, \varepsilon, k}(t, x)=v^{+}\left(T, x_{0}\right)+\frac{\left|x-x_{0}\right|^{2}}{\eta}+k(T-t) .
$$

Since $U, V$ are compact and $b, \sigma$ are continuous, for some $k$ large enough (but no smaller than $\varepsilon / 2 \eta$ ), we have that

$$
-\varphi_{t}^{\varepsilon, \eta, k}-H^{+}\left(t, x, \varphi_{x}^{\varepsilon, \eta, k}, \varphi_{x x}^{\varepsilon, \eta, k}\right)>0 \text { on } \overline{B\left(T, x_{0}, \varepsilon\right)} .
$$

From (77) we obtain $\varphi^{\varepsilon, \eta, k} \geq \varepsilon+w$ on $\mathbb{T}$. Also,

$$
\varphi^{\varepsilon, \eta, k}(T, x) \geq v^{+}\left(T, x_{0}\right) \geq g(x)+\varepsilon \text { for }\left|x-x_{0}\right| \leq \varepsilon .
$$

We now choose $\delta<\varepsilon$ and define

$$
v^{\varepsilon, \eta, k, \delta}=\left\{\begin{array}{l}
w \wedge\left(\varphi^{\varepsilon, \eta, k}-\delta\right) \text { on } \overline{B\left(T, x_{0}, \varepsilon\right)} \\
w \text { outside } \overline{B\left(T, x_{0}, \varepsilon\right)}
\end{array}\right.
$$

Using again the ideas in part 1.1 of the proof, we can show that $v^{\varepsilon, \eta, k, \delta} \in \mathcal{U}^{+}$but $v^{\varepsilon, \eta, k, \delta}\left(T, x_{0}\right)=$ $v^{+}\left(T, x_{0}\right)-\delta<v^{+}\left(T, x_{0}\right)$, leading to a contradiction. Therefore, $v^{+}(T, \cdot) \leq g$.

\section{2. $v^{-}$is viscosity super-solution of upper Isaacs}

2. 1. The interior super-solution property for $v^{-}$: Let $\left(t_{0}, x_{0}\right) \in[0, T) \times \mathbb{R}^{d}$ in the parabolic interior such that a smooth function $\varphi$ strictly touches $v^{-}$from below at $\left(t_{0}, x_{0}\right)$. Assume, by contradiction, that $\varphi_{t}+H^{+}\left(t, x, \varphi_{x}, \varphi_{x x}\right)>0$ at $\left(t_{0}, x_{0}\right)$. There exists a $\varepsilon>0$ and there exists a measurable function $h: V \rightarrow U$, such that

$$
\varphi_{t}\left(t_{0}, x_{0}\right)+\left[b\left(t_{0}, x_{0}, h(v), v\right) \cdot \varphi_{x}\left(t_{0}, x_{0}\right)+\frac{1}{2} \operatorname{Tr}\left(\sigma\left(t_{0}, x_{0}, h(v), v\right) \sigma\left(t_{0}, x_{0}, h(v), v\right)^{T} \varphi_{x x}\left(t_{0}, x_{0}\right)\right)\right]>\varepsilon .
$$

In order to construct the function $h$ above, one does not need to use measurable selection arguments. Using uniform continuity on balls, one can actually choose a finitely valued $h$. Since $\varphi_{t}+b \cdot \varphi_{x}+$ $\frac{1}{2} \operatorname{Tr}\left(\sigma \sigma^{T} \varphi_{x x}\right)$ is continuous in $(t, x, u, v)$ and $U, V$ are compact, it is therefore uniformly continuous on $C \times U \times V$, where $C$ is any compact neighborhood of $\left(t_{0}, x_{0}\right)$. Therefore, we can choose a smaller $\varepsilon>0$ such that (despite the fact that $h$ is only measurable, and maybe not continuous)

$\varphi_{t}(t, x)+\left[b(t, x, h(v), v) \cdot \varphi_{x}(t, x)+\frac{1}{2} \operatorname{Tr}\left(\sigma(t, x, h(v), v) \sigma(t, x, h(v), v)^{T} \varphi_{x x}(t, x)\right)\right]>\varepsilon$, on $B\left(t_{0}, x_{0}, \varepsilon\right) \times V$.

To be precise, small balls here are again intersected with the parabolic interior $[0, T) \times \mathbb{R}^{d}$. On the compact torus $\mathbb{T}=\overline{B\left(t_{0}, x_{0}, \varepsilon\right)}-B\left(t_{0}, x_{0}, \varepsilon / 2\right)$ we have that $\varphi<v^{-}$and the max of $\varphi-v^{-}$is attained, 
therefore it is strictly negative. In other words $\varphi+\eta<v^{-}$on $\mathbb{T}$ for some $\eta>0$. Since we can construct an increasing sequence of stochastic sub-solutions $w_{n} \nearrow v^{-}$, the same Dini type argument similar to [BS14] and [BS13] shows that, for $n$ large enough we have $\varphi+\eta / 2<w_{n}$ on $\mathbb{T}$. For simplicity, fix such an $n$ and call $w=w_{n}$. Now, define, for small $\delta<<\eta / 2$

$$
w^{\delta} \triangleq\left\{\begin{array}{l}
(\varphi+\delta) \vee w \text { on } B\left(t_{0}, x_{0}, \varepsilon\right) \\
w \text { outside } B\left(t_{0}, x_{0}, \varepsilon\right)
\end{array}\right.
$$

Since $w^{\delta}\left(t_{0}, x_{0}\right)>v^{+}\left(t_{0}, x_{0}\right)$, we obtain a contradiction if we can show $w^{\delta} \in \mathcal{U}^{-}$. In order to do so, fix $s$ and let $\tau: C([s, T]) \rightarrow[s, T]$ be a stopping time strategy for the initial time $s$. Fix also $v \in \mathcal{V}(s, s)$. We need to construct an elementary strategy $\tilde{u} \in \mathcal{U}(s, \tau)$ in the Definition of stochastic sub-solutions for $w^{\delta}$, depending on the $v$ we just fixed. This can be again done as follows: since $w$ is a stochastic sub-solution of upper Isaacs, there exists an elementary strategy $\tilde{u}_{1}$ for $w$ starting at $\tau$ and depending on $v$ that does the job. Then

1. if $\varphi+\delta>w$ at $\tau$, follow the constant action $h(v)$.

2. if $w \geq \varphi+\delta$ at $\tau$ follow the strategy $\tilde{u}_{1}$

3. follow the strategy in 1-2 until the first time $\tau_{1}$ when $\left(t, X_{t}\right) \in \partial B\left(t_{0}, x_{0}, \varepsilon / 2\right)$. On this boundary, $w^{\delta}=w$

4. after this, follow the strategy $\tilde{u}_{3} \in \mathcal{U}\left(s, \tau_{1}\right)$ corresponding to the stochastic sub-solution $w$ with starting stopping rule $\tau_{1}$ and second player's strategy $v \in \mathcal{V}(s, s)$.

In a rigorous notation though, we first define $\tilde{u}_{2} \in \mathcal{U}(s, \tau)$ by

$$
\tilde{u}_{2}(t, y(\cdot))=h\left(v(t, y(\cdot)) 1_{\{\varphi(\tau(y), y(\tau(y)))+\delta>w(\tau(y), y(\tau(y)))\}}+\tilde{u}_{1}(t, y(\cdot)) 1_{\{\varphi(\tau(y), y(\tau(y)))+\delta \leq w(\tau(y), y(\tau(y)))\}} \cdot\right.
$$

The key observation here, is that, since $h$ simply maps $V$ into $U$, then, indeed, $\tilde{u}_{2}$ is an elementary strategy $\tilde{u}_{2} \in \mathcal{U}(s, \tau)$. Denote now by $\tau_{1}: C([s, T]) \rightarrow[s, T], \tau \leq \tau_{1} \leq T$,

$$
\tau_{1}(y)=\inf _{\tau(y) \leq t \leq T}\left\{(t, y(t)) \in \partial B\left(t_{0}, x_{0}, \varepsilon / 2\right)\right\} .
$$

Now, if $\tilde{u}_{3} \in \mathcal{U}\left(s, \tau_{1}\right)$ is the strategy defining the stochastic super-solution $w$, corresponding to $v \in$ $\mathcal{V}(s, s)$ then we can define

$$
\tilde{u}=\tilde{u}_{2} \otimes_{\tau_{1}} \tilde{u}_{3} \in \mathcal{U}(s, \tau)
$$

to finish the proof. To actually check that $\tilde{u}$ (depending on $v$ and $\tau$ ) fulfills the condition in the definition of stochastic sub-solution for upper Isaacs, one has to follow similar arguments to the above part 1.1 of the proof, based on Itô's Lemma applied to $d(\varphi+\delta)\left(t, X_{t}\right)$, together with conditioning.

2.2. The terminal condition property for $v^{-}$: the proof is similar to the part 2.1 of the proof, but with an analytic construction very similar to the terminal condition part 1.2. More precisely, first we argue by contradiction, similar to the above step 1.2, then we construct a strategy $\tilde{u}$ as in 2.1 above depending on the fixed $v$ and $\tau$. Finally, we once again use on Itô's Lemma and conditioning in a similar manner to finish the proof.

\section{Verification by Comparison and Dynamic Programming Principle}

Theorem 4.1 Under the standing assumptions, we have

1. $v^{-}=V^{+}=v^{+}$is the unique continuous viscosity solution of the upper Isaacs equation. In addition, the upper value $V^{+}$satisfies the (DPP)

$$
V^{+}(s, x)=\inf _{v \in \mathcal{V}(s, s)} \sup _{u \in \mathcal{U}(s, s)} \mathbb{E}\left[V^{+}\left(\rho\left(X_{.}^{s, x, u, v}\right), X_{\rho\left(X^{s, x, u, v}\right)}^{s, x, u, v}\right], \quad \forall \rho \in \mathbb{B}^{s} .\right.
$$


2. $w^{-}=V^{-}=w^{+}$is the unique continuous viscosity solution of the lower Isaacs equation. In addition, the lower value $V^{-}$satisfies the (DPP)

$$
V^{-}(s, x)=\sup _{u \in \mathcal{U}(s, s)} \inf _{v \in \mathcal{V}(s, s)} \mathbb{E}\left[V^{-}\left(\rho\left(X^{s, x, u, v}\right), X_{\rho\left(X^{s, x, u, v}\right)}^{s, x, u, v}\right], \quad \forall \rho \in \mathbb{B}^{s} .\right.
$$

If the Isaacs condition $H^{-}=H^{+}$holds then the game has a value and the value

$$
w^{-}=V^{-}=V=V^{+}=v^{+}
$$

is the unique continuous viscosity solution of the (unified) Isaacs equation. For each $\varepsilon>0$, there exist $\varepsilon$-saddle point strategies $(u, v) \in \mathcal{U}(s, s) \times \mathcal{V}(s, s)$.

We note that the DPP is a statement about stopping rules $\rho \in \mathbb{B}^{s}$ and is not formulated with respect to stopping times $\rho^{\prime}$ on the original probability space $\left(\Omega, \mathcal{F}, \mathbb{F}^{s}, \mathbb{P}\right)$.

Remark 4.2 In case the Isaacs condition holds, then one only needs to prove half of the Theorem 3.5 (and arguably the easier one), namely that $v^{+}$is a viscosity sub-solution of the Isaacs equation and that $w^{-}$is a super-solution of the very same equation. These statements are perfectly symmetric (and this is the reason why we skipped completely the proof for $w^{+}$and $\left.w^{-}\right)$. In this case, we already have that $w^{-} \leq V^{-} \leq V^{+}-\leq v^{+}$and the comparison argument closes the proof (including the DPP).

Proof: the (DPP) is an easy conclusion of the way we defined $v^{+}, v^{-}, w^{+}$and $w^{-}$, since the stochastic sub/super-solutions of upper/lower Isaacs satisfy the corresponding half DPP. In light of Theorem [3.5, there is basically nothing left to prove, once a comparison result for bounded semicontinuous viscosity solutions of the two Isaacs equations is available. We make it available below to conclude $\diamond$

The following Lemma is basically borrowed from the analysis literature. One can either take it from [CIL92] (with modifications to allow for unbounded domains, bounded semi-continuous solutions and comparison up to $t=0$ ) or (but still up to details) from [Pha09].

Lemma 4.3 Assume b, $\sigma$ are continuous, locally uniformly Lipschitz in $x$, satisfy linear growth (standing assumptions) and $U, V$ are compact. Then

1. for each $R>0$ there exists some $\omega_{R}:[0, \infty) \rightarrow[0, \infty)$, with $\omega_{R}(0+)=0$ such that for any $X, Y$ satisfying

$$
-3 \alpha\left(\begin{array}{cc}
I & 0 \\
0 & I
\end{array}\right) \leq\left(\begin{array}{cc}
X & 0 \\
0 & -Y
\end{array}\right) \leq 3 \alpha\left(\begin{array}{cc}
I & -I \\
-I & I
\end{array}\right)
$$

we have

$L^{u, v}(t, y, \alpha(x-y), Y)-L^{u, v}(t, x, \alpha(x-y), X) \leq \omega_{R}\left(\alpha|x-y|^{2}+|x-y|\right), \forall t, u, v$ and $|x|,|y| \leq R$

with the notation $L^{u, v}(t, x, p, M) \triangleq-b(t, x, u, v) \cdot p-\frac{1}{2} \operatorname{Tr}\left(\sigma(t, x, u, v) \sigma^{T}(t, x, u, v) M\right)$

2. we have that, whenever condition (8) is satisfied,

$$
F(t, y, \alpha(x-y), Y)-F(t, x, \alpha(x-y), X) \leq \omega_{R}\left(\alpha|x-y|^{2}+|x-y|\right), \forall t, u, v \text { and }|x|,|y| \leq R
$$

for $F=-H^{+}$or $F=-H^{-}$

3. consider $v$ a bounded $U S C$ (on $\left.[0, T] \times \mathbb{R}^{d}\right)$ viscosity sub-solution of

$$
-v_{t}+F\left(t, x, v_{x}, v_{x x}\right) \leq 0 \text { on }[0, T) \times \mathbb{R}^{d}
$$

and $w$ a bounded $L S C$ (on $\left.[0, T] \times \mathbb{R}^{d}\right)$ viscosity super-solution of

$$
-w_{t}+F\left(t, x, w_{x}, w_{x x}\right) \geq 0 \text { on }[0, T) \times \mathbb{R}^{d},
$$

where (either) $F=-H^{+}\left(\right.$or $\left.F=-H^{-}\right)$. If $v(T, \cdot) \leq w(T, \cdot)$ then $v \leq w$. 
Proof:

1. condition (9) (denoted by (3.14) in CIL92) is actually proved in CIL92 on page 19, in the elliptic case (no dependence on $t$ ), but the same proof applies to the parabolic case. One should have in mind that the local Lipschitz constant is uniform in $u, v$, therefore, the same function $\omega_{R}$ can be chosen to work for all $u, v$

2. as still mentioned on page 19 in [CIL92, once (9) is uniform in $u, v$, it applies to any sup inf or inf sup transformation, obtaining the nonlinear structural condition (10) (called, to recall, (3.14) in [CIL92]) subject to (8).

3. here, the proof is an adaptation of the comparison result in CIL92 to the case of an unbounded domain and (bounded) semi-continuous solutions, under the structural condition (10) subject to (8) and with the additional important piece of information that coefficients have global linear growth. Comparison is obtained up to time $t=0$, as in [Pha09] or [DFO11]. The case we have here does not fit perfectly in the existing literature, so that we cannot simply quote a single theorem, but the sequence of arguments used is well understood in the viscosity solution literature (see, for example, [DFO11] or [Pha09. We present the key arguments below.

To summarize, we use the penalization in the text-book [Pha09], taking advantage of the linear growth of the coefficients and the special structure of the HJB equation to reduce the problem to a bounded domain, and then appeal to a modification of the results in CIL92 based on the arguments in DFO11] or [Pha09, to obtain comparison up to $t=0$ under the structural condition (3.14) in CIL92.

Assume, for example, that $F=-H^{+}$. Let $\phi(t, x)=e^{-\lambda t}\left(1+|x|^{2}\right)$. From the linear growth assumption LG we have

$$
\sup _{u \in U} \sup _{v \in V}\{|b(t, x, u, v)|+|\sigma(t, x, u, v)|\} \leq C(1+|x|), \quad \forall 0 \leq t \leq T, x \in \mathbb{R}^{d} .
$$

This means that, for $\lambda>0$ large enough, we have

$$
\phi_{t}(t, x)+\sup _{u \in U} \sup _{v \in V}\left[b(t, x, u, v) \cdot \phi_{x}(t, x)+\frac{1}{2} \operatorname{Tr}\left(\sigma(t, x, u, v) \sigma^{T}(t, x, u, v) \phi_{x x}(t, x)\right)\right]<0,
$$

for all $0 \leq t \leq T, x \in \mathbb{R}^{d}$. Therefore, for any $\varepsilon>0$, the LSC function $w^{\varepsilon}=w+\varepsilon \phi$ is (easy to check) a viscosity super-solution of $w_{t}^{\varepsilon}+H^{+}\left(t, x, w_{x}^{\varepsilon}, w_{x x}^{\varepsilon}\right) \leq 0$. According to Theorem 8.2 in CIL92] (actually the follow up observations on page 52), because $F=-H^{+}$satisfies the structural condition (10) subject to (8), we have, for any bounded open domain $\mathcal{O}=\{|x|<$ $R\} \subset \mathbb{R}^{d}$, a comparison result after time $t=0$

$$
v(t, x) \leq w^{\varepsilon}(t, x)+\max \left(\sup _{(0, T] \times \partial \mathcal{O}}\left(v-w^{\varepsilon}\right)^{+}, \sup _{\{T\} \times \overline{\mathcal{O}}}\left(v-w^{\varepsilon}\right)^{+}\right) \quad \forall 0<t \leq T, x \in \mathcal{O} .
$$

However, it is well known (for example from [DFO11], or [Pha09]), that, as long as the viscosity semi-solution property holds at time $t=0$ as well (which is the case here), the doubling argument can be reproduced to provide comparison up to time $t=0$, i.e.

$$
v(t, x) \leq w^{\varepsilon}(t, x)+\max \left(\sup _{[0, T] \times \partial \mathcal{O}}\left(v-w^{\varepsilon}\right)^{+}, \sup _{\{T\} \times \overline{\mathcal{O}}}\left(v-w^{\varepsilon}\right)^{+}\right) \quad \forall 0 \leq t \leq T, x \in \mathcal{O} .
$$

If one does not want to use the version of the Theorem of Sums (TOS) in [DFO11] to get comparison up to $t=0$ in the relation above, an alternative way is to simply define the whole game starting at an earlier negative time. Since both $v, w$ are bounded, the choice of $\phi$ ensures 
that, for large enough domain $\mathcal{O}=\{|x|<R\}$ we have $\sup _{[0, T] \times \partial \mathcal{O}}\left(v-w^{\varepsilon}\right)^{+}=0$. In addition, since $v(T, \cdot) \leq w(T, \cdot) \leq w^{\varepsilon}(T, \cdot)$, we conclude that $\sup _{\{T\} \times \overline{\mathcal{O}}}\left(v-w^{\varepsilon}\right)^{+}=0$ as well. In summary, for each $\varepsilon>0$ we have

$$
v(t, x) \leq w(t, x)+\varepsilon \phi(t, x), \forall 0 \leq t \leq T,|x|<R
$$

for any $R$ large enough (depending on $\varepsilon$ ). We first let $R \nearrow \infty$ and then $\varepsilon \searrow 0$ to conclude that $v \leq w$ on $[0, T] \times \mathbb{R}^{d}$.

\section{Conclusions}

Using Stochastic Perron's method we show that the values of differential games formulated symmetrically over elementary feed-back strategies are (the unique continuous) viscosity solutions of the corresponding Isaacs equations and the DPP holds. We allow for the state equation to be degenerate and time-dependent. Some of the assumptions (for example, compactness of $U, V$ ) seem to be removable, but one still needs enough to get comparison of semi-continuous viscosity solutions.

The program we propose amounts to verification for non-smooth viscosity solutions for the game in the original strong definition, and seems rather elementary compared to either the verification approach in [Świ96b] or proving (parts of) the DPP working with the value functions as in [FS89, Kat95 or CR09. We introduce the class of Elementary (feed-back) Strategies, which have strong intuition behind and are easily amenable to analysis. In particular, a player in a game that is believed to be continuous in time, is most likely to use Elementary Strategies to approximate the saddle point, if such exists. Obviously, one does not expect a saddle point to consist of elementary strategies, but, if the Isaacs condition holds, one has $\varepsilon$-saddle points among elementary strategies. The present work sets the foundation for the analysis (in [S13]) of more realistic zero-sum differential games over mixed (elementary) feed-back strategies. Such a game always has a value, which is the unique solution of the (mixed) Isaacs equation.

We believe the present analysis sheds more light on the connection between controlled diffusions and viscosity solutions of fully non-linear PDE's. One important observation is that, in this symmetric formulation of the game over feed-back strategies, the DPP is expected to hold only over stopping rules, i.e. stopping times where the decision to stop is based upon observing only the state, and not the noise. The technical parts of the proofs (which are basically elementary) are reminiscent of the previous proofs in [BS12, [BS14] and especially [BS13, and, for the analytic constructions, resemble the classic work of Ishii [Ish87. An important part of the contribution resides in the symmetric formulation of the game over elementary strategies (and strong solutions of the state equation) and in the proper (non-trivial) identification of stochastic semi-solutions to the Isaacs equations, that allows for the Stochastic Perron's Method to be completed. With this in mind, we reiterate the conjecture that any stochastic optimization problem could be treated using Stochastic Perron's Method, provided that it is properly formulated, and the stochastic semi-solutions defined accordingly.

\section{References}

[BL08] R. Buckdahn and J. Li, Stochastic differential games and viscosity solutions of HamiltonJacobi-Bellman-Isaacs equations, SIAM Journal on Control and Optimization 47 (2008), no. $1,444-475$.

[BS12] E. Bayraktar and M. Sîrbu, Stochastic Perron's method and verification without smoothness using viscosity comparison: the linear case, Proceedings of the American Mathematical Society 140 (2012), 3645-3654.

[BS13] _ Stochastic Perron's method for Hamilton-Jacobi-Bellman equations, SIAM Journal on Control and Optimization 51 (2013), no. 6, 4274-4294. 
[BS14]_ Stochastic Perron's method and verification without smoothness using viscosity comparison: obstacle problems and Dynkin games, Proceedings of the American Mathematical Society 142 (2014), no. 4, 1399-1412.

[CIL92] M. Crandall, H. Ishii, and P.-L. Lions, User's guide to viscosity solutions of second-order partial differential equations, Bull. Amer. Math. Soc 27 (1992), 1-67.

[CR09] P. Cardaliaguet and C. Rainer, Stochastic differential games with asymmetric information, Applied Math. and Opt. 59 (2009), 1-36.

[DFO11] J. Diehl, P. K. Friz, and H. Oberhauser, Parabolic comparison revisited and applications, preprint, http://arxiv.org/abs/1102.5774, 2011.

[EK72] R. J. Elliott and N. J. Kalton, Values in differential games, Bull. Amer. Math. Soc 72 (1972), no. $3,427-431$.

[EKH03] N. El-Karoui and S. Hamadene, BSDEs and risk-sensitive control, zero-sum and nonzerosum game problems of stochastic functional differential equations, Stoch. Proc. Appl. 107 (2003), no. 1, 145-169.

[FS89] W. H. Fleming and P. E. Souganidis, On the existence of value functions of two-player, zero-sum stochastic differential games, Indiana University Mathematics Journal 38 (1989), no. 2, 293-314.

[HL95] S. Hamadene and J.P. Lepeltier, Zero-sum stochastic differential games and backward equations, Systems and Control Letters 24 (1995), 259-263.

[Isa65]Ｒ. Isaacs, Differential games, John Wiley and Sons, 1965.

[Ish87] H. Ishii, Perron's method for Hamilton-Jacobi equations, Duke Mathematical Journal 55 (1987), no. 2, 369-384.

[Kat95] Markos A. Katsoulakis, A representation formula and regularizing properties for viscosity solutions of second-order fully nonlinear degenerate parabolic equations, Nonlinear Anal. $\mathbf{2 4}$ (1995), no. 2, 147-158. MR 1312585 (95m:35039)

[Kry09] N. V. Krylov, Controlled diffusion processes, Stochastic Modelling and Applied Probability, vol. 14, Springer-Verlag, Berlin, 2009, Translated from the 1977 Russian original by A. B. Aries, Reprint of the 1980 edition.

[Kry13a] _ On the dynamic programming principle for uniformly nondegenerate stochastic differential games in domains, Stoch. Proc. Appl. 123 (2013), no. 8, 3273-3298.

[Kry13b] N.V. Krylov, On the dynamic programming principle for uniformly nondegenerate stochastic differential games in domains and the Isaacs equations, Probab. Theory Relat. Fields (2013), published electronically.

[KS88] N. N. Krasovskiı and A. I. Subbotin, Game-theoretical control problems, Springer Series in Soviet Mathematics, Springer-Verlag, New York, 1988, Translated from the Russian by Samuel Kotz. MR 918771 (89b:90248)

[KS01] I. Karatzas and W. Sudderth, The controller-and-stopper game for a linear diffusion, Annals of Probability 29 (2001), no. 3, 1111-1127.

[LS85] P.-L. Lions and P. E. Souganidis, Differential games, optimal control and directional derivatives of viscosity solutions of Bellman's and Isaacs' equations, SIAM J. Control Optim. 23 (1985), no. 4, 566-583. MR 791888 (87c:49038) 
[Nis88] Makiko Nisio, Stochastic differential games and viscosity solutions of Isaacs equations, Nagoya Math. J. 110 (1988), 163-184. MR 945913 (90b:93100)

[Pha09] Huyên Pham, Continuous-time stochastic control and optimization with financial applications, Stochastic Modelling and Applied Probability, vol. 61, Springer-Verlag, Berlin, 2009.

[PZ12] T. Pham and J. Zhang, Two person zero-sum game in weak formulation and path dependent Bellman-Isaacs equation, preprint, 2012.

[S1̂1] M. Sîrbu, On martingale problems with continuous-time mixing and values of zero-sum games without Isaacs conditions, preprint, http://arxiv.org/abs/1307.4686, 2013.

[Świ96a] A. Święch, Sub- and superoptimality principles of dynamic programming revisited, Nonlinear Anal. 26 (1996), no. 8, 1429-1436. MR 1377672 (97b:49026)

[Świ96b] Andrzej Święch, Another approach to the existence of value functions of stochastic differential games, J. Math. Anal. Appl. 204 (1996), no. 3, 884-897. MR 1422779 (97j:90091) 